\title{
Ansiedade e desempenho escolar no ensino fundamental I
}

\author{
Anxiety and performance school in basic education
}

Neide de Brito Cunha*

Universidade do Vale do Sapucaí

Sandra Maria da Silva Sales

Oliveira**

Universidade do Vale do Sapucaí
Thaísa Vilhena Silva***

Universidade do Vale do Sapucaí

Antônio José Figueiredo Oliveira****

Universidade do Vale do Sapucaí

Resumo Este estudo objetivou avaliar a ansiedade em alunos do Ensino Fundamental I e eventuais diferenças com relação ao sexo, idade, ano escolar, tipo de escola e desempenho acadêmico nas disciplinas de matemática e português. Nesta pesquisa de campo, foram avaliados 134 alunos de duas escolas, uma pública e outra particular do sul do Estado de Minas Gerais. Do total, 70 eram meninas e as idades variaram de 7 a 10 anos. Os estudantes estavam cursando do $2^{\circ}$ ao $5^{\circ}$ ano. Foi utilizado o Inventário de Ansiedade na Escola e as notas dos alunos nas disciplinas de Português e Matemática. Os resultados apontaram um nível médio de ansiedade escolar e não foram encontradas diferenças estatisticamente significativas na avaliação da ansiedade com relação às variáveis estudadas. Como há carência de pesquisas com as crianças quanto à ansiedade escolar nesse nível de ensino, considera-se importante avaliar outras amostras.

PALAVRAS-CHAVE: Ansiedade; Desempenho acadêmico; Ensino fundamental.

\begin{abstract}
This study aimed to evaluate anxiety in students of elementary school and any differences with regard to gender, age, school year, school type and academic performance in math and Portuguese. In this field research, we evaluated 134 students from two schools, one public and one particular of the southern state of Minas Gerais-Brasil. Of the total, 70 were girls and the ages ranged from 7 to 10 years. The students were attending the 2 nd to 5 th year. Anxiety Inventory at the School and students' grades in the subjects of Portuguese and Mathematics was used. The results showed an average level of school anxiety and statistically significant differences weren't found in the evaluation of anxiety regarding the variables studied. As there is a lack of research with children as the school anxiety in this level of education, it is considered important to evaluate other samples.
\end{abstract}

KEYWORDS: Anxiety; Academic performance; Elementary school. 


\section{Introdução}

A ansiedade é o sentimento que acompanha um estado geral de perigo, advertindo as pessoas de que há algo a ser temido no futuro. Refere-se a um sentimento de inquietação que pode traduzir-se em manifestações de ordem fisiológica, como agitação, movimentos precipitados, hiperatividade e de ordem cognitiva, como atenção e vigilância redobrada a determinados aspectos do meio, pensamentos de possíveis desgraças etc.

Essas manifestações podem estar associadas a acontecimentos ou situações de natureza passageira, chamada de ansiedade estado ou constituir uma maneira estável e permanente de reagir, provavelmente com base na própria constituição individual, a chamada ansiedade traço. Os transtornos de ansiedade podem também interferir diretamente em processos de atenção e provocar prejuízos de aprendizagem e memória (LANDEIRA-FERNANDEZ; CRUZ; BRANDÃO, 2007).

Segundo a American Psychological Association (APA, 2000), os transtornos de ansiedade apresentam quatro grandes grupos de sinais e sintomas. São eles: reações somáticas ou fisiológicas, incluindo taquicardia, sudorese ou tontura; reações cognitivas; reações emocionais, como irritação, desespero, excesso de preocupação e de responsabilidade; alterações comportamentais, como o retraimento social ou a diminuição no rendimento escolar ou profissional.

A ansiedade para a criança é um sentimento desagradável, pois por não se conhecer tão bem como o adulto, ela não consegue descrever este sentimento ou mesmo identificá-lo, tanto como exagerado ou anormal, não percebendo que existe algo de errado consigo mesma. No entanto, deve-se salientar que a ansiedade não é um fator negativo para o ser humano, porém quando exposto a uma situação de desconhecimento ou de difícil controle, a mesma pode tomar grandes proporções, levando a reações imprevisíveis (BROUCO, 2006).

Entende-se por transtornos de ansiedade padrões de resposta exagerada a situações de estresse de média intensidade, e ocorrem mais comumente em indivíduos com uma predisposição neurobiológica, o que os torna facilmente ansiosos. A ansiedade patológica é percebida pela sua severidade, persistência, associação a eventos neutros e prejuízo significativo no funcionamento e desenvolvimento psicossocial da criança ou adolescente (SERRA, 1989).

Os transtornos de ansiedade estão entre as doenças psiquiátricas mais comuns em crianças e adolescentes, com prevalência de $4 \%$ a $20 \%$ dessa população. Essa variação no percentual pode ser justificada por conta da aplicação de diferentes métodos de avaliações e instrumentos (ASBAHR, 2004; PETERSEN, 2011).

Em relação ao sexo, Ito et al. (2008) consideram que a probabilidade de os transtornos ansiosos é a mesma tanto para o sexo feminino quanto para o masculino. Porém, os autores verificaram que, excetuando-se as fobias específicas, os transtornos pós-traumáticos e transtorno do pânico são mais predominantes em pessoas do sexo feminino. 
Siegel (1954) já demonstrava uma relação entre as dificuldades escolares e os problemas emocionais desde a primeira definição dos transtornos de aprendizagem. De acordo com Nelson e Hardwood (2011), essa relação vem sendo assumida naturalmente, pelo fato de se entender que os alunos com problemas escolares experimentam mais dificuldades emocionais que os alunos que não as possuem. No entanto, os autores ressaltam que são escassos os estudos empíricos que fazem uso de métodos quantitativos e análises estatísticas que apoiam a associação entre alta ansiedade e problemas de aprendizagem.

No Brasil, a condução de estudos nessa área é escassa e estes podem ser relevantes para o âmbito da educação e da psicologia educacional (BZUNECK, 1991; COSTA; BORUCHOVITCH, 2004; OLIVEIRA, 2012). Acrescenta-se a isso o fato de o ensino fundamental ser um período importante na vida escolar dos alunos e abranger a infância e o início da adolescência, compreendendo uma fase da vida marcada por transformações que poderão ser importantes nos comportamentos e êxitos futuros.

$\mathrm{Na}$ fase escolar, a ansiedade pode ocorrer por uma série de situações. $\mathrm{O}$ aluno está predisposto às relações interpessoais com adultos e crianças, bem como a eventos sociais que requerem certa capacidade de adaptação, como as situações sociais (BEIDEL; TURNER; MORRIS, 1999; OGUNDOKUN, 2011). Os alunos desenvolvem atitudes para atender às expectativas externas, buscando a aceitação social (CASTILLO et al., 2000).

Assim, o funcionamento social da criança na escola também pode indicar sintomas de ansiedade. Alunos extremadamente ansiosos evitam a interação com os pares o se comportam socialmente de forma menos competente em diversas possibilidades de interação e exposição (WOOD, 2006).

As situações avaliativas também são propensas a produzir altos níveis de ansiedade. Nesse sentido, Birenbaum e Pinku (1997) relatam que a ansiedade frente às provas escolares pode ser observada muito antes, na véspera e na situação de prova, nos estudantes com medo de fracassar. A criação de crenças errôneas quanto ao próprio desempenho escolar também gera ansiedade.

Assim, a ansiedade no contexto escolar pode se manifestar de várias formas, inclusive com a irrupção de fatores fisiológicos que afetam o funcionamento cognitivo necessário para aprendizagem dos alunos, bem como o bom desempenho escolar (EYSENCK et al., 2007; PEREIRA; BARROS; MENDONÇA, 2012). Por exemplo, Wood (2006) verificou redução da ansiedade em 40 alunos com alta ansiedade e idades compreendidas entre seis e treze anos que participaram de um programa de intervenção cognitivo comportamental. Esses estudantes apresentaram melhoras em seu desempenho escolar e no funcionamento social.

Mychailyszyn, Mendez e Kendall (2010) investigaram o comportamento de estudantes de sete a quatorze anos que apresentavam transtorno de ansiedade de separação, fobia social e desordem de ansiedade generalizada diagnosticados em situação clínica e compararam o ajustamento escolar desse grupo com outro de estudantes 
sem diagnóstico de ansiedade. $\mathrm{O}$ estudo considerou o desempenho acadêmico como um dos fatores de ajustamento na escola. Os resultados indicaram que os alunos com elevados níveis de ansiedade demostraram consideravelmente maiores prejuízos em termos de adequação de comportamentos na escola e desempenho acadêmico que os sem transtorno de ansiedade.

Drahota et al. (2013) pesquisaram habilidades do dia a dia em grupos de crianças em idade escolar divididos em crianças com transtornos de ansiedade e crianças sem transtornos de ansiedade. Os resultados indicaram que os transtornos ansiosos estão correlacionados com menor domínio dessas habilidades. Além disso, os pesquisadores concluíram que crianças com mais idade são mais afetadas pelos transtornos ansiosos em comparação às crianças mais novas.

No contexto brasileiro, Costa e Boruchovitch (2004) desenvolveram um estudo para verificar as relações entre a ansiedade e o uso de estratégias de aprendizagem em estudantes com idades entre seis e dezoito anos. Os dados sobre as estratégias de aprendizagem foram coletados mediante uma entrevista individual estruturada, constituída por 16 perguntas abertas e fechadas relativas às estratégias de aprendizagem que foram traduzidas e adaptadas de Self-Regulated Learning Interview Schedule por Boruchovitch (1995). O inventário de ansiedade traço e estado/forma infantil (IDATE) foi utilizado como instrumento para a coleta dos dados relativos a ansiedade. Os resultados apontaram para um maior nível de ansiedade associado a um conhecimento deficiente sobre estratégias de administração do tempo de estudo visando somente à preparação para uma prova. Ao contrário do que era esperado pelas autoras, não foram encontradas relações significativas entre a ansiedade e estratégias de realização de provas dos participantes.

Oliveira (2012), com o uso do Inventário de Ansiedade na Escola (OLIVEIRA; SISTO, 2002), comparou o nível de ansiedade em um grupo de alunos com desempenho escolar adequado com um grupo de alunos que foram indicados pela diretora da escola por apresentarem baixo rendimento acadêmico constante. Os resultados indicaram que o grupo de alunos com baixo desempenho foi significativamente mais ansioso que o grupo de alunos sem problemas de desempenho.

Fernandes e Silveira (2012) realizaram uma pesquisa no contexto acadêmico do ensino fundamental para verificar os níveis de ansiedade e as possíveis relações entre a ansiedade acadêmica e a motivação de estudantes com idades compreendidas entre sete e onze anos. Os resultados apontaram que os alunos apresentaram índices abaixo da média de ansiedade para a pontuação total da escala e pontuações moderadas de motivação acadêmica. No entanto, foram observadas correlações estatisticamente significativas e positivas entre a ansiedade e a motivação extrínseca.

Uma pesquisa que envolveu 559 alunos do ensino fundamental de escolas públicas e particulares brasileiras, com idades compreendidas entre 8 e 18 anos, com o objetivo de investigar possíveis diferenças em relação ao gênero e idade, utilizou o Inventário de ansiedade na escola. Os resultados demonstraram maior pontuação no sexo masculino em medo genérico, situações avaliativas e ansiedade total. Em relação 
à faixa etária, alunos mais velhos apresentaram maior pontuação nos fatores de insatisfação e evitação, enquanto que os alunos mais novos, com até 10 anos apresentaram pontuações menores em relação aos outros alunos (JOLY; OLIVEIRA, 2012).

Fassis, Mendes e Carmo (2014) investigaram, por meio de dois estudos, aspectos relacionados à ansiedade Matemática em alunos do ensino fundamental. No Estudo 1, 770 estudantes responderam a uma escala de ansiedade à Matemática e foram identificadas pequenas diferenças entre graus de ansiedade matemática e conduziram-se comparações entre gênero, período, rede de ensino e série. Não houve diferenças significativas entre gênero, rede de ensino e série, porém alunos do período vespertino apresentaram maior ansiedade. No Estudo 2, os autores verificaram a relação entre nenhuma/baixa e alta/extrema ansiedade e desempenho em Matemática em oito alunos que participaram do Estudo 1, divididos em baixa ansiedade, em um grupo, e alta/extrema ansiedade em outro grupo. Os alunos com maior ansiedade foram os que apresentaram notas mais altas quando comparados com os alunos que apresentaram baixa ansiedade.

Com base nas investigações apresentadas, considerou-se a importância de dar continuidade na investigação da ansiedade de alunos do ensino fundamental. Considerou-se, também, a relevância de investigar variáveis relativas ao contexto e aos participantes. Nesse sentido, os objetivos estabelecidos nesta pesquisa foram: avaliar a ansiedade em alunos do Ensino Fundamental I e eventuais diferenças com relação ao sexo, idade, ano escolar, tipo de escola e desempenho acadêmico nas disciplinas de matemática e português.

\section{Método}

Nesta pesquisa de campo, delineou-se um estudo de levantamento com vistas à análise de variáveis relativas ao contexto e aos participantes. Foram utilizadas estatísticas descritivas e inferenciais não paramétricas, visto que o teste de Shapiro -Wilk $(p<0,001)$ revelou a ausência da distribuição normal dos dados.

\section{Participantes}

Participaram 134 alunos de duas escolas, uma pública $(\mathrm{n}=48)$ e outra particular $(\mathrm{n}=86)$ do sul do Estado de Minas Gerais. Do total, 70 eram meninas e as idades variaram de 7 a 10 anos, com média de 8,58 e desvio padrão 1,02. Os estudantes estavam cursando o $2^{\circ}(n=23,17,2 \%), 3^{\circ}(n=48,35,8 \%), 4^{\circ}(n=36,26,9 \%)$ e $5^{\circ}(n=27$, 20,1\%) anos do Ensino Fundamental I.

\section{Instrumentos}

\section{Inventário de Ansiedade na Escola - IAE (OLIVEIRA; SISTO, 2002)}

Foi construído com itens baseados nos sintomas de ansiedade descritos no DSM IV, na CID 10 e em revisão da literatura (do ano de 1960 ao ano 2000) acerca da temática ansiedade em situações escolares. Contém 34 itens, é uma escala de tipo Li- 
kert com três opções de resposta: sempre ( 2 pontos), às vezes (1 ponto) e nunca (zero). As frases se agrupam em quatro fatores: Fator 1, medo genérico - com 16 itens; Fator 2, satisfação ou compensação - com 8 itens; Fator 3, evitação - com 4 itens; Fator 4, medo de situações avaliativas - com 6 itens. Para se obter a pontuação de ansiedade deve-se somar os itens dos Fatores 1, 3 e 4, assim o intervalo de pontos pode variar de 0 até 52. Os itens do Fator 2 não são considerados para a análise por se tratarem de itens de compensação que não avaliam a ansiedade na escola, mas que compensam as situações negativas representadas pelos outros fatores. São itens relacionados com situações que expressam gosto e satisfação para as crianças no contexto escolar.

Como exemplos de itens do instrumento, pode-se citar: "Fico com medo de errar os exercícios"; "Se tenho que ir à lousa, meus músculos ficam tensos"; "Não consigo me concentrar nas atividades escolares"; "Sinto falta de ar na sala de aula"; "Durante o tempo que fico na escola meu coração bate rápido".

As medidas de precisão foram calculadas com base na consistência interna para cada subescala e para o instrumento como um todo. Dentre as subescalas, a que apresentou menor precisão por alfa foi a do Fator 2 (satisfação ou compensação) com coeficiente de 0,66. No Fator 3 (evitação) o coeficiente alfa é 0,68 e no fator 4 (medo de situações avaliativas) alfa igual a 0,69. O Fator 1 (medo genérico) apresentou coeficiente alfa de 0,88 . Esses valores podem ser considerados bons ao se considerar o número de itens. Para a ansiedade geral observa-se coeficiente alfa de 0,84.

\section{Notas dos alunos nas disciplinas de Português e Matemática}

As notas obtidas corresponderam ao período compreendido pelo primeiro bimestre, visto que a coleta de dados se deu no início do segundo bimestre de 2015. A escolha por essas disciplinas deveu-se ao fato de elas serem as que mais similaridades tinham entre as escolas em carga horária. Verificou-se que uma ou outra disciplina era ministrada em uma escola e em outra não. Assim decidiu-se pelas disciplinas consideradas também por sua importância nas avaliações em larga escala. Na escola pública, as notas foram obtidas na sua secretaria e eram classificadas em conceitos com as letras A, B e C. Já na escola particular, as notas dos alunos correspondiam a valores entre $0 \mathrm{e}$ 25 pontos. Desse modo, para fazer a equivalência dos diferentes conceitos das escolas, às crianças que obtiveram de 0 a 8 (de 0 a $33 \%$ de 25 pontos) foi atribuído o valor 1 , para as que obtiveram de 9 a 16 (de 34 a 66\%) foi atribuído o valor 2 e para as crianças que obtiveram de 17 a 25 (de 67 a 100\%), o valor 3. Do mesmo modo, para a escola pública foi realizada a equivalência dos conceitos para os valores $(A=1),(B=2)$ e $(C=3)$.

\section{Procedimentos}

O projeto foi submetido e aprovado pelo Comitê de Ética, sob Protocolo CAAE: 25177913.5.0000.5102. Em seguida, houve autorização da Secretaria de Educação de cada cidade onde foi realizada a coleta e as diretoras das escolas foram contatadas e os detalhes da pesquisa foram combinados. Os participantes foram assegurados de que a pesquisa não influenciaria na nota, não afetaria o seu desempenho acadêmico na escola, além de ser de caráter estritamente confidencial. Os pais dos 
alunos foram livres para deixarem os filhos participarem ou não da pesquisa. Aos que aceitaram participar foram entregues os Termos de Consentimento Livre e Esclarecido para assinatura.

A aplicação dos instrumentos foi feita coletivamente com as crianças em suas salas de aula, em horários cedidos pelos professores. Primeiramente, os alunos assinaram os Termos de Assentimento, concordando em participar da pesquisa. Em seguida, os alunos foram respondendo uma a uma cada questão que era lida pelos pesquisadores responsáveis pela coleta dos dados.

\section{Resultados}

De acordo com o primeiro objetivo, recorreu-se à estatística descritiva para identificar média, desvio padrão, mínimo e máximo das respostas obtidas de ansiedade nos fatores e total. Os resultados estão ilustrados na Tabela 1.

Tabela 1 - Estatística descritiva da frequência de ansiedade por fatores e total percebida pelos alunos

\begin{tabular}{lcccc}
\hline & Média & Desvio padrão & Mínimo & Máximo \\
\hline Medo genérico & 16,83 & 3,21 & 9 & 26 \\
Evitação & 6,07 & 1,73 & 2 & 12 \\
Medo avaliação & 6,19 & 1,62 & 2 & 10 \\
Ansiedade total & 29,08 & 4,58 & 18 & 44 \\
\hline
\end{tabular}

Observa-se, na Tabela 1 , que a maior média $(16,83)$ foi obtida no fator "Medo genérico", no entanto, cabe lembrar que este fator reúne o maior número de itens $(16)$ em relação aos demais fatores. A menor média $(6,07)$ foi obtida para o fator "Evitação", também com o menor desvio padrão $(\mathrm{DP}=1,62)$. Para a "Ansiedade total" a média foi de 29,08, pouco acima da metade dos pontos para o total da escala que é de 26 pontos.

Para alcançar o segundo objetivo, foi realizado o teste de Kruskal Wallis para a comparação da IAE quanto ao sexo dos participantes, no entanto, os resultados não apontaram diferenças significativas, embora o posto de média dos meninos $(\mathrm{M}=71,38)$ tenha sido maior que o das meninas $(\mathrm{M}=63,96)$. Também foi realizado esse teste para comparação quanto às idades das crianças, que variou de 7 a 10 anos, de acordo com o terceiro objetivo, e mais uma vez não houve diferenças significativas, com apenas um resultado marginalmente significativo para o "Medo genérico" $(\mathrm{p}=0,05)$.

Quanto ao quarto objetivo, de identificar diferenças pelo ano escolar, o teste de Kruskal Wallis também não revelou diferenças estatisticamente significativas quanto à ansiedade. Também foram verificados os resultados quanto às escolas e os resultados também não indicaram índices significativos, embora as médias dos postos 
Neide de Brito Cunha - Sandra Maria da Silva Sales Oliveira - Thaísa Vilhena Silva Antônio José Figueiredo Oliveira

dos alunos da escola pública $(M=72,31)$, tenham sido mais altas que as das crianças da escola particular $(M=64,81)$.

Com vistas a identificar a existência ou não de diferenças significativas com relação aos grupos contrastantes (valores 1 e 3), na disciplina de matemática, adotou-se o teste de Kruskal Wallis para a comparação da IAE. Os valores obtidos estão apresentados na Tabela 2.

Tabela 2 - Comparação entre os grupos contrastantes na disciplina de matemática pela prova de Kruskal-Wallis

\begin{tabular}{lccccc}
\hline & Grupos & N & Média dos ranks & $X^{2}$ & $p$ \\
\hline Medo genérico & 1 & 7 & 43,79 & 306,50 & 0,609 \\
& 3 & 97 & 49,41 & 4446,50 & \\
Evitação & 1 & 7 & 48,29 & 338,00 & 0,943 \\
\multirow{2}{*}{ Medo avaliação } & 3 & 97 & 49,06 & 4415,00 & \\
& 1 & 7 & 35,50 & 248,50 & 0,179 \\
Ansiedade total & 3 & 97 & 50,05 & 4504,50 & \\
& 1 & 7 & 42,64 & 298,50 & 0,534 \\
& 3 & 97 & 49,49 & 4454,50 & \\
\hline
\end{tabular}

Não houve diferença significativa quanto às médias dos postos das subescalas conforme visto na Tabela 2, para a disciplina de matemática. O mesmo procedimento foi elaborado com relação aos grupos contrastantes (valores 1 e 3 ) na disciplina de português, como mostrado na Tabela 3 .

Tabela 3 - Comparação entre os grupos contrastantes na disciplina de português pela prova de Kruskal-Wallis

\begin{tabular}{lccccc}
\hline & Grupos & N & Média dos ranks & $X^{2}$ & $p$ \\
\hline Medo genérico & 1 & 7 & 46,93 & 328,50 & \\
\multirow{2}{*}{ Evitação } & 3 & 101 & 55,02 & 5557,50 & 0,506 \\
Medo avaliação & 1 & 7 & 64,71 & 453,00 & \\
& 3 & 101 & 53,79 & 5433,00 & 0,364 \\
Ansiedade total & 1 & 7 & 51,93 & 363,50 & \\
& 3 & 101 & 54,68 & 5522,50 & 0,819 \\
& 1 & 7 & 57,43 & 402,00 & \\
& 3 & 101 & 54,30 & 5484,00 & 0,797 \\
\hline
\end{tabular}

Conforme observa-se na Tabela 3, os resultados também não revelaram diferenças estatisticamente significativas para os fatores e para a Ansiedade Total na 
disciplina de português. Nota-se que tanto em matemática como em português há um pequeno número de participantes no grupo $1(n=7)$. Verificou-se que esses sujeitos eram todos da escola pública e que cinco deles pertenciam ao grupo 1 tanto na disciplina de matemática como na de português.

\section{Discussão}

De acordo com os objetivos deste estudo, foi medida a ansiedade das crianças e verificadas eventuais diferenças com relação a variáveis contextuais como sexo, idade, ano escolar, tipo de escola e desempenho acadêmico nas disciplinas de matemática e português. Os resultados apontaram um nível médio de ansiedade escolar, indicando que no contexto acadêmico das escolas pesquisadas, embora houvesse a perspectiva do surgimento de condições novas e desafiadoras que podem produzir ansiedade, ela não se manifestou de forma patológica (ASBAHR, 2004; MYCHAILYSZYN, MENDEZ; KENDALL, 2010; SERRA, 1989). Pode-se inferir que as manifestações de ansiedade apresentadas que se caracterizaram na amostra deste estudo foram as de natureza passageira, ou seja, a de ansiedade estado, que não provocam prejuízos para a aprendizagem e memória (LANDEIRA-FERNANDEZ; CRUZ; BRANDÃO, 2007).

Cabe considerar, no entanto, que as crianças deste estudo ainda se encontram no começo da vida acadêmica e nessa idade ainda é difícil descrever a ansiedade, pois o aluno ainda não percebe se há algo de errado consigo mesmo (BROUCO, 2006). Além disso, as pesquisas levantadas demonstram que as crianças maiores e os adolescentes costumam ser mais acometidos pela ansiedade escolar (DRAHOTA et al., 2013).

Não foram encontradas diferenças significativas com relação ao sexo, embora a média dos postos dos meninos tenha sido superior. Esse resultado vai ao encontro das afirmações de Ito et al. (2008), de que a probabilidade de transtornos ansiosos é a mesma para ambos os sexos. Da mesma maneira, não houve diferença quanto ao ano escolar, o que pode ser corroborado pela questão das idades das crianças, de acordo com as explicações acima.

$\mathrm{O}$ ano escolar também não apontou diferenças significativas, mesmo com a média mais alta para ansiedade para os alunos da escola pública. $\mathrm{O}$ mesmo aconteceu com relação ao tipo de escola, embora as médias mais altas tenham sido as das crianças da escola particular. Também não foram encontradas diferenças estatisticamente significativas quanto ao desempenho acadêmico nas disciplinas de matemática e português. Esses resultados corroboram os do estudo de Fassis, Mendes e Carmo (2014), que investigaram aspectos relacionados à ansiedade matemática em alunos do ensino fundamental. Esses autores encontraram apenas pequenas diferenças entre graus de ansiedade matemática nas comparações entre gênero, período, rede de ensino e série. No entanto, no seu segundo estudo, com oito alunos divididos em baixa ansiedade, em um grupo, e alta/extrema ansiedade em outro grupo, encontraram maior ansiedade nos que apresentaram notas mais altas quando comparados com os alunos que apresentaram baixa ansiedade. 
A relação entre dificuldades escolares e problemas emocionais vem sendo assumida naturalmente por se entender que os alunos com problemas escolares experimentam mais dificuldades emocionais que os alunos que não as possuem (NELSON; HARWOOD, 2011). No entanto, os autores já ressaltavam a escassez de estudos empíricos com uso de métodos quantitativos, o que se constatou também no levantamento de pesquisas deste trabalho.

Há que considerar que a ansiedade pode atrapalhar tanto os alunos que têm êxito no contexto escolar como os que não têm. Assim, caberia ao professor administrar a ansiedade dos alunos e contribuir para a redução da ansiedade em sala de aula mediante certos procedimentos, como: prover os alunos com instruções sobre formas adequadas de estudo; possibilitar oportunidades para que alunos ansiosos possam falar em pequenos grupos ou responder a perguntas com respostas curtas (como sim ou não); usar atividades que envolvam aprendizagem cooperativa; esclarecer o objetivo das provas; evitar pressões de tempo nas situações de exames; determinar um espaço de tempo que assegure que todos os alunos consigam completar a prova; variar os tipos de avaliação, entre outros. Cabe lembrar que a ansiedade não é um fator negativo para o ser humano, desde que ela não tome grandes proporções (BROUCO, 2006). No entanto ela aparece entre as doenças psiquiátricas mais comuns em crianças e adolescentes (ASBAHR, 2004; PETERSEN, 2011).

\section{Considerações finais}

Não foram encontradas, neste estudo, diferenças estatisticamente significativas na avaliação da ansiedade em alunos do ensino fundamental com relação ao sexo, idade, ano escolar, tipo de escola e desempenho acadêmico nas disciplinas de matemática e português. Considerou-se importante essa avaliação por conta do pequeno número de publicações sobre o tema por meio de estudo quantitativo e em alunos desse nível de ensino, pois são encontradas mais pesquisas com universitários com relação à ansiedade no contexto escolar.

A ansiedade se refere a uma reação natural do organismo, ou seja, um extinto desenvolvido de sobrevivência para que o ser humano reaja diante do perigo. No entanto, quando excede o nível de normalidade traz grande desconforto em determinadas situações da vida dos indivíduos. Os resultados desta pesquisa apontaram índices médios de ansiedade escolar mesmo diante da perspectiva de que o contexto escolar é uma parte fundamental da vida da criança, em que ela se desenvolve social, cognitiva e emocionalmente, sendo assim um lugar propício para o surgimento de condições novas e desafiadoras que podem produzir ansiedade.

A literatura levantada enfatiza que a ansiedade pode atrapalhar tanto o aluno que se saem bem quanto os que não. Assim, isso refere-se ao trabalho do professor que tem como tarefa administrar a ansiedade dos alunos e contribuir muito para a redução da ansiedade em sala de aula mediante certos procedimentos, como: prover os alunos com instruções sobre formas adequadas de estudo; possibilitar oportunidades para que alunos ansiosos possam falar em pequenos grupos ou responder a perguntas com respostas curtas (como sim ou não); usar atividades que envolvam aprendizagem 
cooperativa; esclarecer o objetivo das provas; evitar pressões de tempo nas situações de exames; determinar um espaço de tempo que assegure que todos os alunos consigam completar a prova; variar os tipos de avaliação, entre outros.

As escolas dos alunos aqui avaliados provavelmente investem na formação de seus professores. A formação docente tem que ser pensada como um aprendizado profissional ao longo da vida, o que implica envolvimento dos professores em processos intencionais e planejados, se preocupando e investindo com aspectos emocionais de seus alunos, além dos cognitivos.

Cabe destacar que este estudo contou com um número pequeno de participantes e que foram contempladas somente duas disciplinas, as mais avaliadas nas avaliações em larga escala no país e nos Estados. Como já foi apontado, há carência de pesquisas com as crianças quanto à ansiedade escolar, assim espera-se que mais trabalhos levem em consideração esse nível de ensino e que possam contemplar mais disciplinas da grade curricular.

\section{Referências}

American Psychiatric Association (APA). Manual Diagnóstico e Estatístico de Transtornos Mentais (DSM - IV-TR). 4 ed. Porto Alegre/RS: Artes Médicas, 2000.

ASBAHR, F. R. Transtornos ansiosos na infância e adolescência: aspectos clínicos e neurobiológicos. Jornal de Pediatria, v. 80, n. 2, p. 28-34, 2004.

BEIDEL, D. C., TURNER, S. M.; MORRIS, T. L. Psychopathology of childhood social phobia. Journal of American Academy of Child and Adolescent Psychiatry, v. 38, n. 6, p. 643-650, 1999.

BIRENBAUM, M.; PINKU, P. Effects of test anxiety, information Organization and testing situation on performance in two test formats. Contemporary Educational Psychology, v. 22, p. 23-38, 1997.

BORUCHOVITCH, E. A identificação e o estudo das variáveis associadas ao fracasso escolar brasileiro. Projeto de pesquisa realizado na qualidade de bolsista de recém doutor do CNPq. Faculdade de Educação, Departamento de Psicologia Educacional, Universidade de Campinas, 1995.

BROUCO, G. R. A influência da brincadeira atenuando a ansiedade escolar em período pré-avaliativo. In: X ENFEFE - Encontro Fluminense de Educação Física Escolar. Niteroi-RJ, 08 e 09 de dezembro, 2006. Disponível em: <http://www.uff.br/gef/logo-pos-grad_enfefe.htm>. Acesso em: 29 abr. 2016.

BZUNECK, J. A. Ansiedade e desempenho numa prova de matemática: Um estudo com adolescentes. In: II Simpósio interdisciplinar de estudos cognitivos, Laboratório de Estudos Cognitivos da Universidade Estadual de Londrina, Londrina, PR/Brazil. 1991.

CASTILLO, A. et al. Transtornos de ansiedade. Revista Brasileira de Psiquiatria, v. 22, n. 2, p. 20-23, 2000.

COSTA, E. R.; BORUCHOVITCH, E. Compreendendo Relações entre Estratégias de Aprendizagem e a Ansiedade de Alunos do Ensino Fundamental de Campinas. Psicologia: Reflexão e Crítica, v. 17, n. 1, p. 15-24, 2004.

DRAHOTA, A. et al. Daily living skills in school-age children with and without anxiety disorders. British Journal of Clinical Psyhology, v. 52, p. 107-112, 2013. 
Neide de Brito Cunha - Sandra Maria da Silva Sales Oliveira - Thaísa Vilhena Silva Antônio José Figueiredo Oliveira

EYSENCK, M. W. et al. Anxiety and cognitive performance: Attentional control theory. Emotion, v. 7, p. 336-353, 2007.

FASSIS, D.; MENDES, A. C.; CARMO, J. S. Diferentes graus de ansiedade à matemática e desempenho escolar no ensino fundamental. Psicologia da Educação, n. 39, p. 4761, 2014. Disponível em: <http://pepsic.bvsalud.org/scielo.php?script=sci_arttext\&pi$\mathrm{d}=\mathrm{S} 1414-69752014000200005 \& \operatorname{lng}=$ pt\&nrm=iso $>$. Acesso em: 19 jun. 2016.

FERNANDES, D. C.; SILVEIRA, M. A. Evaluación de la motivación académica y la ansiedad escolar y posibles relaciones entre ellas. Psico-USF, v. 17, n. 3, p. 447-455, 2012.

ITO, L. M. et al. Terapia cognitivo-comportamental da fobia social. Revista Brasileira de Psiquiatria, v. 30, supl. 2, p. 96-101, 2008.

JOLY, M. C. R. A.; OLIVEIRA, S. M. S. S. Avaliação da Ansiedade Escolar em Alunos do Ensino Fundamental. Revista Psicología Trujillo, v. 14, n. 1, p. 21-30, 2012.

LANDEIRA-FERNANDEZ, J.; CRUZ, A. P. M.; BRANDAO, M. L. Padrões de respostas defensivas de congelamento associados a diferentes transtornos de ansiedade. Psicologia USP, v. 17, n. 4, p. 175-192, 2006. Disponível em <http://www.scielo.br/scielo.php?script=sci_arttext\&pid=S0103-65642006000400010\&lng=pt\&nrm=iso >. Acesso em: 19 jun. 2016.

MYCHAILYSZYN, M. P.; MENDEZ, J. L.; KENDALL, P. C. School Functioning in Youth With and Without Anxiety Disorders: Comparisons by Diagnosis and Comorbidity School. Psychology Review, v. 39, n. 1, p. 106-121, 2010.

NELSON, J. M.; HARWOOD, H. Learning disabilities and anxiety: A meta-analysis.Journal of Learning Disabilities, v. 44, n. 1, p. 3-17, 2011.

OGUNDOKUN, M. O. Learning style, school environment and test anxiety as correlates of learning outcomes among secondary school students. Ife Psychologia, v. 19, p. 321-336, 2011.

OLIVEIRA, S. M. S. S. O Modelo de Rasch para avaliar o Inventário de Ansiedade na Escola. Tese (Doutorado em Psicologia). Universidade São Francisco, Itatiba, 2012.

OLIVEIRA, S. M. S. S.; SISTO, F. F. Estudo para uma escala de ansiedade escolar para crianças. Psicologia Escolar e Educacional, v. 6, n. 1, p. 57-66, 2002.

PEREIRA, A. I. F.; BARROS, L.; MENDONÇA, D. Cognitive errors and anxiety in school aged children. Psicologia: Reflexão e Crítica, v. 25, n. 4, p. 817-823, 2012.

PETERSEN, C. S. Evidências de efetividade e procedimentos básicos para Terapia CognitivoComportamental para crianças com transtornos de ansiedade. Revista Brasileira de Psicoterapia, v. 13, n. 1, p. 39-50, 2011.

SERRA, A. S. L. Medo e ansiedade na adolescência. Psicologia em Curso, v. 1, n. 1, p. 34-46, 1989.

SIEGEL, M. The personality structure of children with reading disabilities as compared with children presenting other clinical problems. Nervous Child, v. 10, p. 409-414, 1954.

WOOD, J. J. Effect of Anxiety Reduction on Children's School Performance and Social Adjustment. Developmental Psychology, v. 42, n. 2, p. 345- 349, 2006.

* Professora Doutora da Universidade do Vale do Sapucaí, Pouso Alegre, Minas Gerais, Brasil.

** Professora Doutora da Universidade do Vale do Sapucaí, Pouso Alegre, Minas Gerais, Brasil.

**** Mestre em Educação pela Universidade do Vale do Sapucaí, Pouso Alegre, Minas Gerais, Brasil.

***** Mestre em Educação pela Universidade do Vale do Sapucaí, Pouso Alegre, Minas Gerais, Brasil. 


\section{Correspondência}

Sandra Maria da Silva Sales Oliveira - Universidade do Vale do Sapucaí. Av. Prefeito Tuany Toled, no 470. Fátima I. CEP: 37550-000. Pouso Alegre, Minas Gerais, Brasil.

E-mail: neidedebritocunha@gmail.com-smsso23@gmail.com-thaisavilhena@hotmail.com-ajfopsico4@ gmail.com

Recebido em 22 de junho de 2016

Aprovado em 02 de junho de 2017 
